\title{
Brachial plexus neuropathy in a gymnast associated with chicken pox
}

\author{
Michael Kennedy, MB and Michael Molloy, MRCP \\ Sports Clinic, Cork Regional Hospital, Wilton, Ireland
}

A case report is presented of brachial plexus neuropathy in an international gymnast in association with chickenpox and the clinical features, investigations and prognosis are discussed.

Keywords: Brachial plexus neuropathy, gymnast, chickenpox

A 14-year-old gymnast presented to our sports clinic complaining of weakness in his right shoulder. Three months earlier he had contracted chicken pox which was followed one week later by the sudden onset of pain in his right shoulder. There was no history of trauma. The pain lasted for three weeks, shortly after which he noticed weakness of his right shoulder requiring cessation of training.

On examination he had wasting of infraspinatus, supraspinatus and serratus anterior muscles with marked winging of his right scapula. He had diminished power of internal rotation, external rotation and abduction of his right shoulder. X-rays of his cervical spine were at that time normal. He was examined by a consultant neurologist and nerve conduction studies and biopsy of the infraspinatus muscle were carried out. This showed chronic partial denervation of muscle with normal motor conduction velocities and decreased amplitude of sensory action potentials suggestive of brachial neuritis. He was treated initially with physiotherapy and exercise programmes.

He was followed-up regularly and returned to training, though not fully recovered after one vear. Because of the absence of full muscle recovery, he had a cervical myelogram to exclude a compression lesion: this was normal. The nerve conduction study was repeated after fourteen months and showed some electrophysiological improvement.

On review two and a half vears after presentation, he had not fully recovered but had adapted his technique sufficiently to compete with distinction at international level.

\section{Discussion}

Brachial plexus neuropathy is a clinical entity usually presenting with sudden pain in the shoulder girdle. In about two weeks muscular weakness follows as the pain diminishes. Later, atrophy is noted'. The cause is unknown and it may be related to either systemic or local infection or to an allergic/hypersensitivity response. Some 15 per cent of cases described have followed vaccination and 15 per cent a viral infection ${ }^{2}$. It may be an allergic response to a stimulus leading to axonal degeneration of the peripheral nerve. Chickenpox has not previously been described as an antecedant. Laboratory investigations do not help in making the diagnosis'.

Brachial plexus neuropathy is twice as frequent in males as in females and occurs more frequently on the right side. The prognosis is good, with 80 per cent recovery after two years, and 90 per cent recovery after three years with anv residual weakness usually being minor'. A poor prognosis is indicated by prolonged or recurrent pain, no improvement after three months, and complete or lower plexus neuropathies ${ }^{3}$.

There is no proven therapeutic gain from systemic or intramuscular steroids in the final outcome though they may relieve pain initially. Muscle exercise programmes and physiotherapy likewise have no effect on the final outcome'.

\section{References}

1 Tsairis, P., Dvck, P.I. and Mulder, D.W. Natural History of Brachial Plexus Neuropathy Arch Neural 1972, 27, 109-117

2 Neuralgic amotrophy - Still a clinical syndrome, Editorial Lancet 1980, 729-730

3 Dyck, P.J., Thomas, P.K., Lambert, E.H. and Bunge, R.P. Peripheral-Neuropathy 2nd Edition

Thanks to Dr R. Galvin, consultant neurologist, Cork Regional Hospital 